\title{
DNA Methyltransferases Directed Anti-Cancerous Plant Medicine (Xanthomicrol and Galloyl) Based Molecular Docking and Dynamics Simulation
}

\author{
Ehsan Heidari Soureshjani ${ }^{1}$, Ali Kazemi Babaheydari2 ${ }^{*}$, Elahe Saberi ${ }^{3}$ \\ ${ }^{1}$ Young Researcher and Elites Club, Islamic Azad University, Shahrekord Branch, Shahrekord, Iran \\ ${ }^{2}$ Faculty of Science, Department of Chemistry, Islamic Azad University, Shahrekord Branch, Shahrekord, Iran \\ ${ }^{3}$ Faculty of Basic Sciences, Department of Biology, Shahrekord Branch, Islamic Azad University, Sahrekord, Iran \\ Email: ${ }^{*}$ kazemiali@iaushk.ac.ir, kazemibali@gmail.com
}

Received 10 May 2015; accepted 27 June 2015; published 30 June 2015

Copyright (C) 2015 by authors and Scientific Research Publishing Inc.

This work is licensed under the Creative Commons Attribution International License (CC BY). http://creativecommons.org/licenses/by/4.0/

(c) (7) Open Access

\begin{abstract}
DNA methyltransferases 1 (DNMT1) has been looked as crucial targets against various types of cancers. MD simulations have advanced to a point where the atomic level information of biological macromolecule (protein or DNA-protein or protein-protein) can easily be advantageous to predict the functionality. In this study we utilize xanthomicrol and galloyl compounds to investigate potential compounds for the inhibition of DNMT1, and the results of these two compounds are compared with drug decitabine. Xanthomicrol and galloyl are found to dock successfully within the active site of DNMT1. A comparison of the inhibitory potential of screened xanthomicrol inhibited DNMT1 approximately is identical with those of their corresponding drugs, decitabine. The stability of the DNMT1 with the best docked xanthomicrol, were further analysed in molecular dynamics (MD) simulation and compared with those of the respective drugs namely decitabine which revealed stabilization of these complexes within $300 \mathrm{~ns}$ of simulation with better stability of DNMT1.
\end{abstract}

\section{Keywords}

DNA Methyltransferases 1, Decitabine, Molecular Dynamics, Galloyl, Xanthomicrol

\section{Introduction}

DNA methylation refers to the addition of a methyl group to one of the four bases that constitute the coding sequence of DNA. In humans, methylation is normally added only on the 5 position of the cytosine base in a *Corresponding author.

How to cite this paper: Soureshjani, E.H., Babaheydari, A.K. and Saberi, E. (2015) DNA Methyltransferases Directed Anti-Cancerous Plant Medicine (Xanthomicrol and Galloyl) Based Molecular Docking and Dynamics Simulation. Computational Molecular Bioscience, 5, 13-19. http://dx.doi.org/10.4236/cmb.2015.52003 
post-DNA synthesis reaction catalyzed by one of several DNA methyltransferases. DNA methylation plays a key role in chromatin structure, suppression of the activity of endogenous parasitic sequences, and stable suppression of gene expression (epigenetic silencing), and a process normally is reserved for special situations such as the inactive X-chromosome and imprinted genes [1]. Epigenetic aberrations are common in the development and progression of cancer cells and are mediated by DNA methyltransferases (DNMTs), histone deacetylases (HDACs) and microRNA (miRNA). These modifications alter gene function and malignant cellular transformation. DNA methylation is a reversible reaction, and catalyzed by three major DNMTs. One is DNMT1, which preserves the methylation patterns throughout each cell division [2] [3]. The others are DNMT3a and DNMT3b, which transfer a methyl group to previously unmethylated genomic regions [4]. Hypermethylation of DNA is a key epigenetic mechanism for the silencing of many genes, including those for cell cycle regulations, receptors, DNA repair, and apoptosis [5]. In this aberrant methylation, the cytosine of the CpG Island in or near the promoter region of the newly synthesized DNA strand is methylated by DNMT. The methylated CpG Island has higher binding affinity to methyl-CpG binding domain proteins that recruit transcriptional corepressors, such as histone deacetylases, resulting in chromosome condensation and transcription repression [6] [7]. The inhibition of DNMT, especially DNMT1, would block the hypermethylation of the newlysynthesized DNA strand, and results in the reversal of the hypermethylation and there-expression of the silenced genes [8]-[10]. The therapeutic activity of three cytosine nucleoside analogues, 5-azacytidine (azacitidine, 5 AC) and 5-aza-20-deoxycytidine (Decitabine, DAC) in acute myeloid leukemia (AML) and myelodysplastic syndromes (MDS) has been the focus of numerous recent studies. When applied at low doses, the compounds reduce genomic DNA methylation as a consequence of their irreversible binding to DNMT after incorporation into newly synthesized DNA [11]. Interference with aberrant DNA methylation, leading to the reactivation of silenced tumor suppressor genes, accounts, at least in part, for the DNMT inhibitors' antitumoral effects [12]. Agents that preferentially inhibit proliferation of neoplastic cells may offer means of developing drugs effectively against cancer in human. One of the strategies for finding such agents is screening plant material, especially those which have been reported to have anti-cancer, anti-inflammatory, anti-fungal or anti-bacterial effects. Spinal-Z is a traditional Iranian anticancer remedy. Spinal-Z is an extract consisting of two plants: Peganum harmala Linn. Seeds and Dracocephalum kotschyii Boiss leaves. The active ingredient in the leaf extract of D. kotschyii appears to be a flavone identified as xanthomicrol. Xanthomicrol is able to inhibit proliferation of a number of malignant cells [13]. Four kinds of galloyl flavonol glycosides are found in the leaf extract of Pemphis acidula, a plant growing on the subtropical seashore. The galloyl flavonoids show more efficient antioxidant activity than that of the corresponding flavonol glycosides [14]. Docking is the process by which two molecules fit together in three-dimensional space and molecular docking is a useful tool in structural molecular biology and computer-assisted drug design, and bioinformatics is seen as an emerging field with the potential to significantly improve how drugs are found and brought to the clinical trials and eventually released to the marketplace. This method is widely used to predict the predominant binding mode(s) oligand with a protein. Besides, reliability in docking of ligand molecules to protein or other targets is an important challenge for molecular modeling. One applications of the docking technique includes the prediction of the binding mode of novel drugs. Docking permits the scientist to virtually screen a database of compounds and bode the strongest binders based on variant scoring functions. The docking analyses are carried by AutoDock (4.2) docking software. In this research, we utilize xanthomicrol and galloyl compounds to investigate potential lead drugs for DNMT1. The database virtual screen provides a rapid search to identify potent compounds for DNMT1 interactions. Molecular dynamics (MD) simulation is used to simulate the dynamic changes between DNMT1 protein and docked ligand.

In this study we utilized computer-aided drug design (CADD) in this research in order to design potential lead drugs for AD therapy. CADD is an efficient approach for the rapid identification of potential lead compounds in target therapy and has been widely used in drug design including virus therapy, cancer therapy, treatment of sleeplessness, neuropathic therapy, weight loss therapy, diabetic treatment, inflammation treatment, erectile dysfunction treatment, and hair loss therapy [15].

\section{Materials and Methods}

\subsection{Molecular Docking}

At first 3D atomic coordinates of DNMT1 (ID: 2QRV) were obtained from the Protein Data bank [16] and prepared for docking. AutoDock (4.2), a similar suite of programs involved genetic algorithm, was employed to 
gain an insight into the compounds (Figure 1) bindings with DNMT1. All nonpolar hydrogens were merged and partial atomic charges were assigned using the Gasteiger-Marsili method. Different grid boxes with different grid points in size with a grid-point spacing of $0.375 \AA$ were considered for docking. Each map was centered such that it covered the entire protein including all possible binding sites.

\subsection{Docking Analysis}

The generated ligands were docked into the defined binding site on the DNMT1protein structure. Ligplot plus was used to analysis docking poses for H-bond and hydrophobic interactions.

\subsection{Molecular Dynamics Simulation}

Then molecular dynamics simulations were performed using the Gromacs 4.5.4 software, under G43a1 force field. Topology files and parameters of small compounds in protein-ligand complexes were generated for GROMACS simulation by SwissParam web server. The calculations were carried out using the crystallographic structure of domain DNMT1. The complex with the best position obtained from docking, was embedded into a box containing SPC216 model water. At first the entire system was minimized using the steepest descent followed by conjugate gradients algorithms, then the solvent and $\mathrm{Na}^{+}, \mathrm{CL}^{-}$were allowed to evolve using minimization and molecular dynamics at NVT ensemble for 500 ps and at NPT ensemble for 1000 ps at $100 \mathrm{~K}$ in which initial configuration of the structures was kept fixed. Finally in order to obtain equilibrium geometry at $300 \mathrm{~K}$ and $1 \mathrm{~atm}, 1 \mathrm{~ns}$ MD simulation run was performed at NPT ensemble. The time step for the simulation was $2 \mathrm{fs}$. Van der Waals and electrostatic forces cutoff were $14 \AA$ and $12 \AA$ respectively.

\section{Results}

\subsection{Docking Analysis}

We used the Autodock (4.2) to select potent two compounds (Xanthomicrol and Galloyl) which have high affinity and almost equal with DNMT1 compared with Decitabine. The results of the docking score are listed in Table 1 and all candidates are ranked by Autodock. We regard the top three compounds candidates, Decitabine, Xanthomicrol, and Galloyl, as potential compounds and further analyze the binding poses in DNMT1 protein structure. Thus, the Xanthomicrol was the most potent for DNMT1 (Eb $=-5.83 \mathrm{kcal} / \mathrm{mol})$, Galloyl was the weakest for DNMT1 $(\mathrm{Eb}=-3.71 \mathrm{kcal} / \mathrm{mol})$. The Xanthomicrol compared to Decitabine was found to be the best compound analyzed showing potent inhibition of DNMT1.

The docking pose of Decitabine revealed two H-bonds with Phe238. For Galloyl, the docked ligand formed H-bonds with Thr 871, Val 873 and Thr 858. In the 2D diagram of docking poses by Ligplot (Figure 2), Decitabine with the surrounding residues, Pro 272, Arg 278, Pro 277, Pro 237, Lys 273, Glu 233, Ala 270 and Arg 271 displayed hydrophobic interactions with the ligand. The 2D diagram of Xanthomicrol was similar to the 3D docking poses, which form two H-bonds with Pro 279; hydrophobic interaction residues included Pro 277, Pro 272, Ala 270, Arg 271, Phe 238, Pro 237, Arg 191 and Asp 239 also external bond with Arg 278. For Galloyl, the 2D diagram is corrected with 3D docking pose that interacted with Met 876, Arg 878, Ser 877, Arg 881, Asp 872, Ser 874, Asn 875, Cys 857 and Arg 881 by hydrophobic forces.

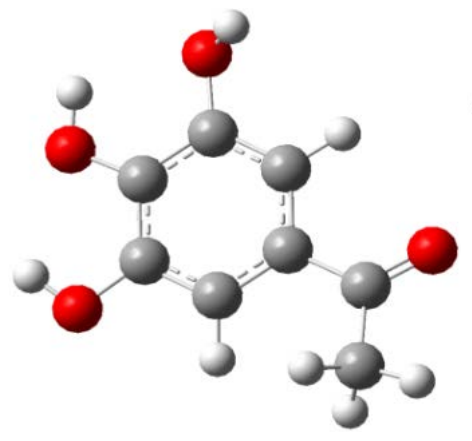

(a)

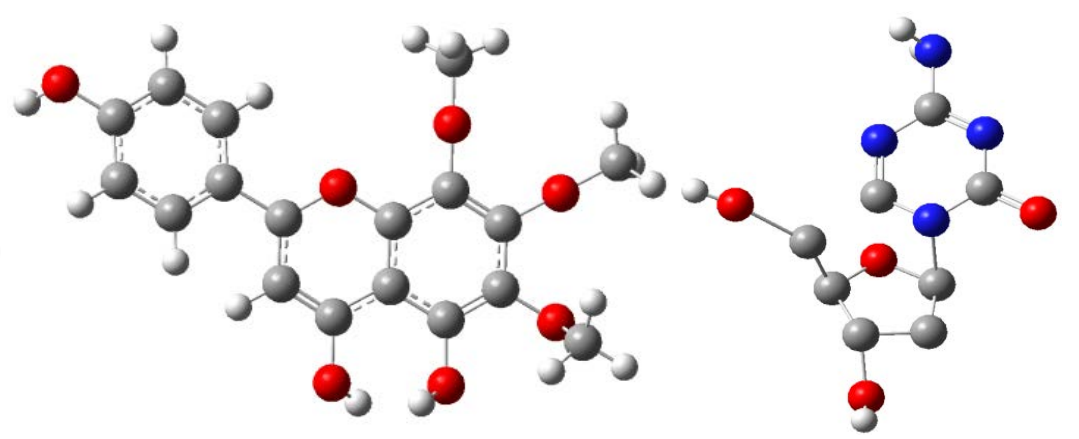

(b) (c)

Figure 1. Chemical scaffold of (a) Galloyl, (b) Xanthomicrol, and (c) Decitabine. 


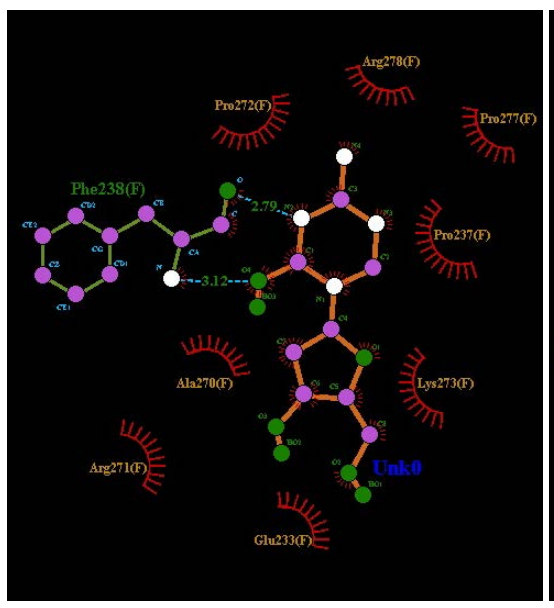

(a)

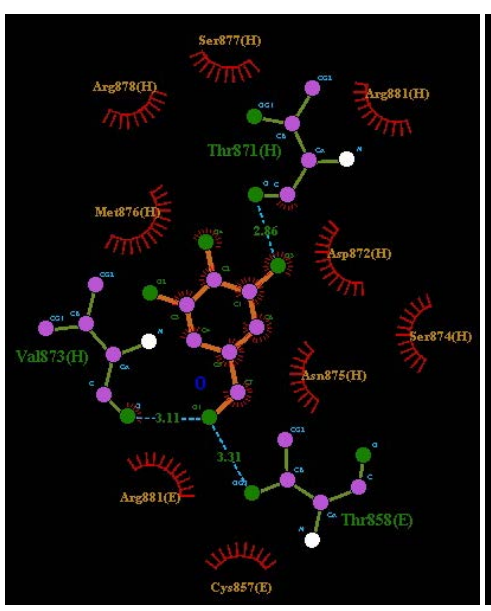

(b)

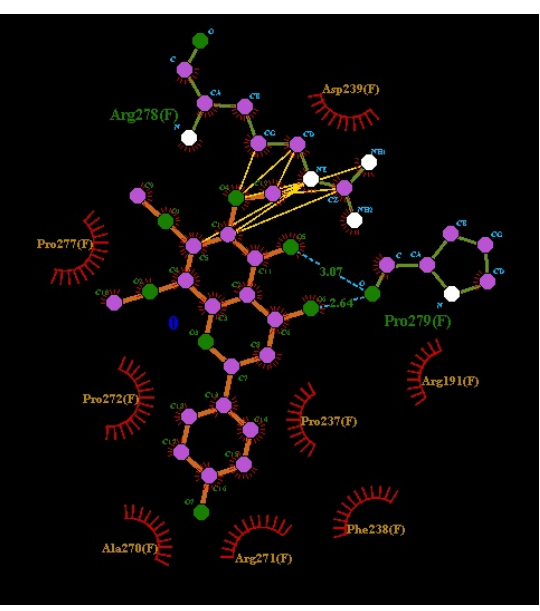

(c)

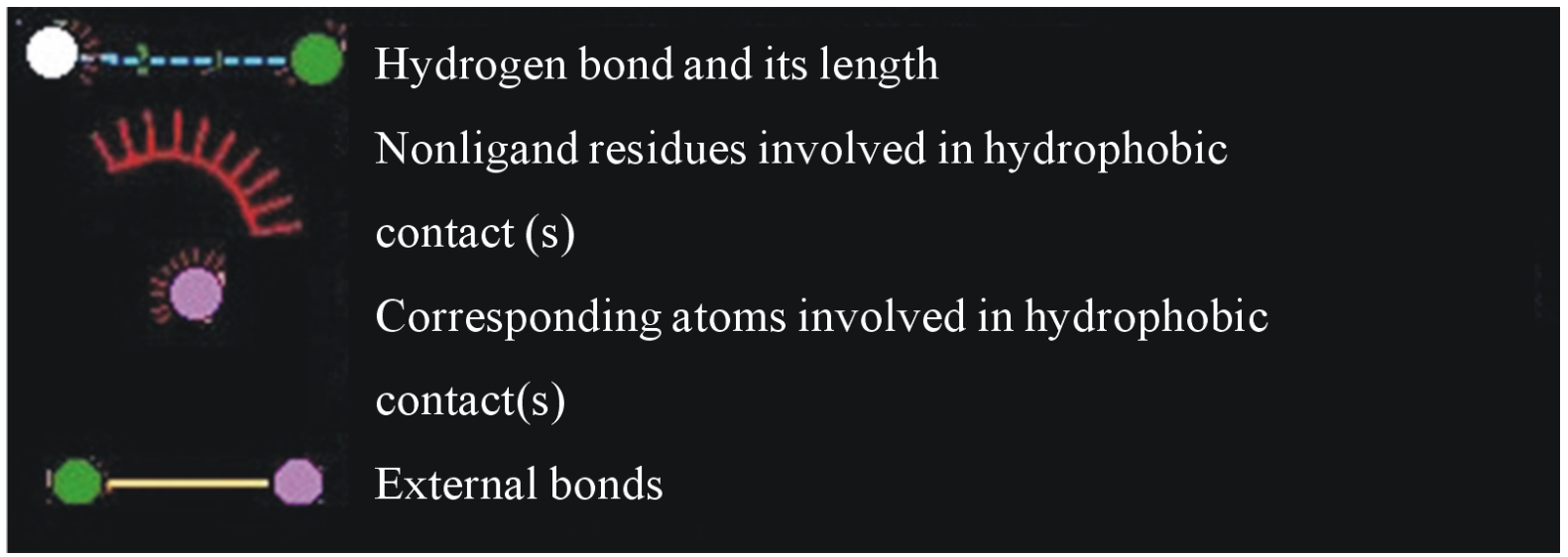

Figure 2. H-bond and hydrophobic analysis of docking poses by Ligplot plus tool for each docked ligand in DNMT1: (a) Decitabine, (b) Xanthomicrol and, and (c) Galloyl.

Table 1. Results interaction DNMT1 with Decitabine, Xanthomicrol and Galloyl conformers by Auto dock.

\begin{tabular}{cccccc}
\hline Compound & Eb (kcal/mol) & $\begin{array}{c}\text { Intermolecular Energy } \\
\mathbf{( k c a l / m o l )}\end{array}$ & $\begin{array}{c}\text { Internal Energy } \\
\mathbf{( k c a l / m o l )}\end{array}$ & $\begin{array}{c}\text { Torsional Energy } \\
\mathbf{( k c a l / m o l )}\end{array}$ & $\begin{array}{c}\text { Unbounded External } \\
\text { Energy (kcal/mol) }\end{array}$ \\
\hline Decitabine & -3.84 & -5.33 & -0.45 & 1.49 & -0.45 \\
Xanthomicrol & -5.83 & -7.32 & -1.59 & 1.49 & -1.59 \\
Galloyl & -3.71 & -5.21 & -0.36 & 1.49 & -0.36 \\
\hline
\end{tabular}

\subsection{Molecular Dynamics Simulation Analyses}

The stability of the DNMT1 during the simulation in the presence of Decitabine, Xanthomicrol and Galloyl was evaluated by the root mean square deviation (RMSD) and by monitoring its secondary structure elements during the simulations. As documented in Figure 3, the backbone RMSD was steady over time, exhibiting fluctuations that reached maximal values of $4.0 \AA$ in the simulation. This consistency dictates that the DNMT1was stable under the simulation conditions.

For total energy analysis, no significantly increased values were observed among all simulation times (Figure 4). The total energy of all systems remained in $-9695000 \mathrm{~kJ} / \mathrm{mol}$. These results suggest that all structures of the complexes tend to become constant after the initial simulation time. In the present study, it may be concluded that that the Xanthomicrol qualifies for its further testing, using in-vivo/in-vitro studies, to develop as a potential DNMT1 directed anti-cancerous drug.

As DNMT1 have one hydrogen bond donor $(\mathrm{OH})$ and one hydrogen bond acceptor $(\mathrm{O})$, the probability to 


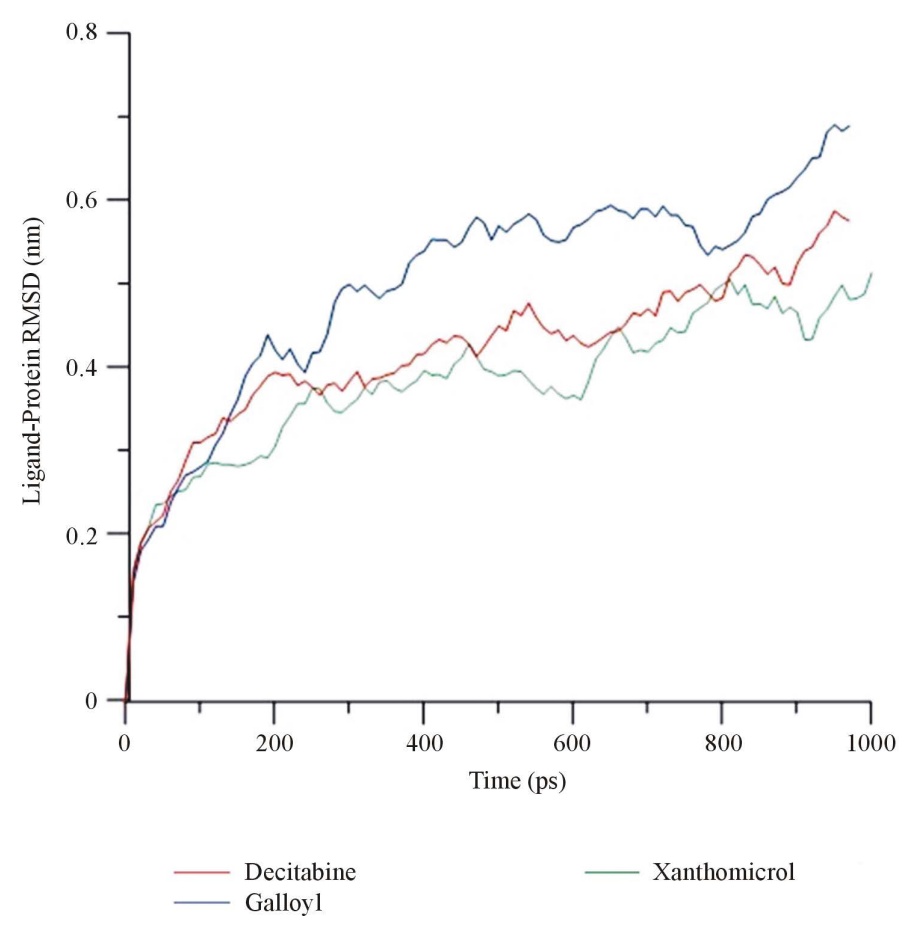

Figure 3. Backbone RMSD In the presence of Decitabine, Xanthomicrol and Galloyl during 1 ns MD simulation.

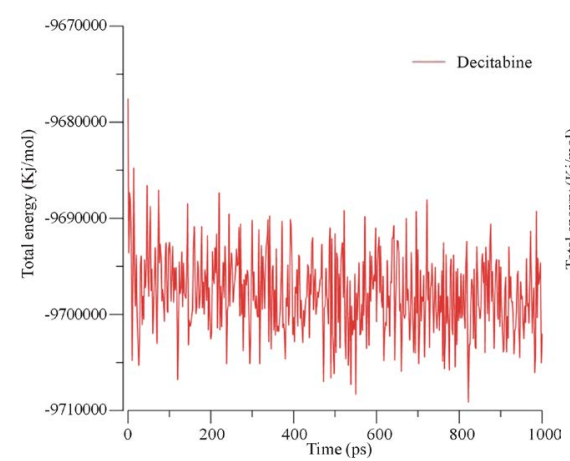

(a)

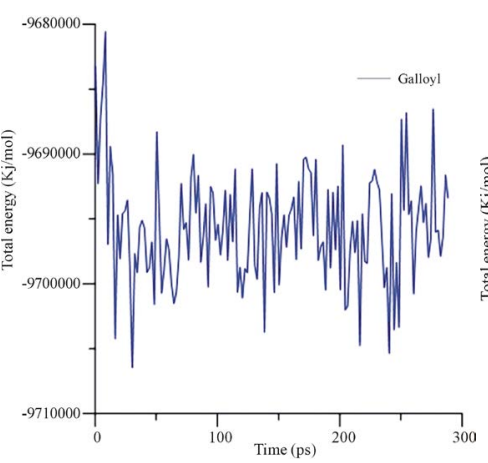

(b)

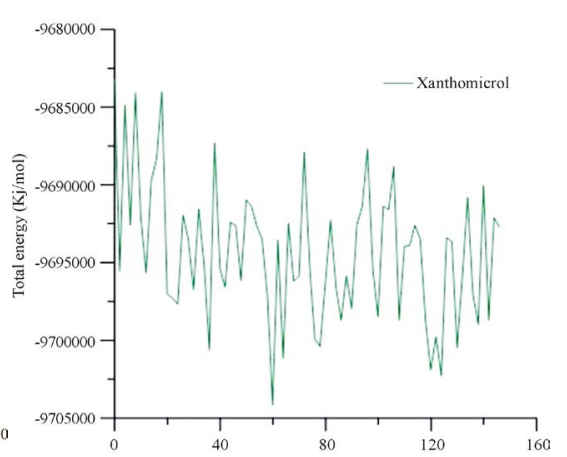

(c)

Figure 4. Total energy of DNA methyltransferases 1 (DNMT1) with docked ligand: (a) Decitabine, (b) Galloyl, and (c) Xanthomicrol 1ns (1000 ps) during MD simulation.

form hydrogen bonds is rare. Number of H-bonds formed between DNMT1 and Decitabine, Xanthomicrol, and Galloyl compounds during a MD simulation was calculated. Of all these three cases, the number of H-bonds fluctuates between 1200 and 1280 (Figuar 5). A change in the number of hydrogen bonds in the simulation is approximately the same for all three compounds.

\section{Conclusion}

DNMT1 plays the key role in cell proliferation phase of all types of developing cancer. Conceptually, targeting DNMT1 will disrupt both DNA replication and transcription, thereby leading to inhibition of cell division and consequently stopping the growth of actively dividing cancerous cells. Therefore, any therapeutic approach targeted against DNA replication in cancerous cells will certainly be a key to the development of a novel anti-cancerous agent. Furthermore, long term use of synthetic drugs such as Decitabine has been reported to develop severe side effects. Therefore, for therapeutic applications, there is a need to identify more specific inhibitors of DNMT1, coming from natural sources, which are relatively safer in use with little or no side effects. In 


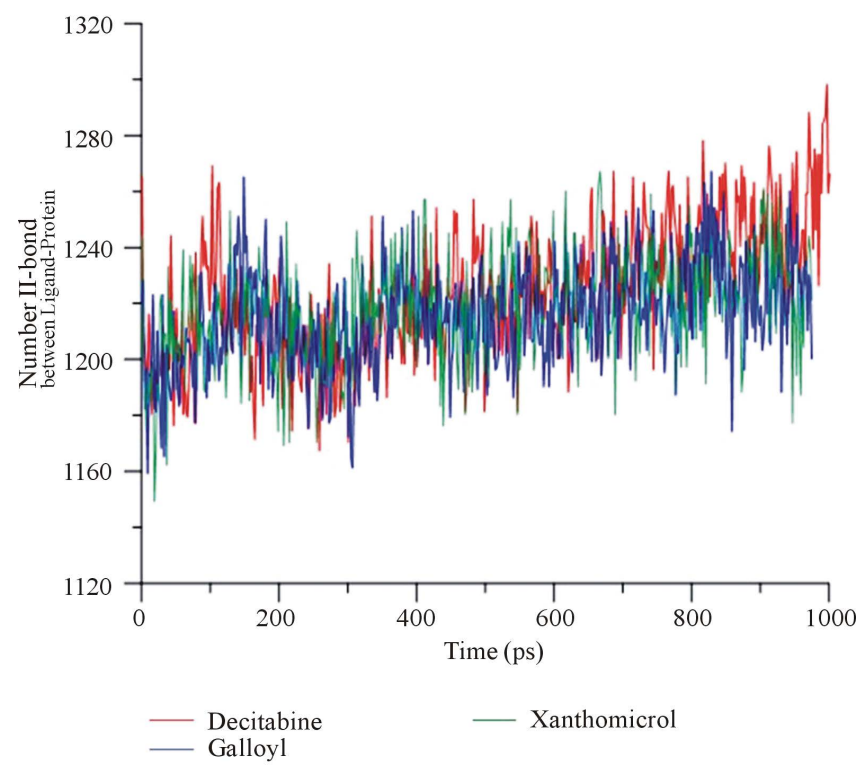

Figure 5. Hydrogen bonds formed between DNMT1 and Decitabine, Xanthomicrol and Galloyl during 1ns (1000 ps) MD simulation.

view of these, in the present study, plant derived one hundred anti-cancerous has been selected and their pharmacokinetic properties are predicted through insilico analyses. Thus Xanthomicrol and Galloyl are selected. Furthermore, docking analyses reveal that Xanthomicrol inhibites DNMT1 more strongly, while Galloyl inhibites preferentially DNMT1 weaker than those of respective drugs, Decitabine outstanding. The results of best docked, Xanthomicrol, are further validated and compared with those of respective drugs namely Decitabine using molecular dynamics (MD) simulation. Thus, the present study makes a foundation for further investigations based on the experimental data (wet lab data) for therapeutic application of screened Plant Medicine in general and Xanthomicrol in particular.

\section{Acknowledgements}

This study was supported by Azad University, Shahrekord Branch, and the Young Research Club of Islamic Azad University, Shahrekord Branch, Shahrekord, Iran.

\section{References}

[1] Bird, A. (2002) DNA Methylation Patterns and Epigenetic Memory. Genes Development, 16, 6-21. http://dx.doi.org/10.1101/gad.947102

[2] Li, E., Bestor, T.H. and Jaenisch, R. (1992) Targeted Mutation of the DNA Methyltransferase Gene Results in Embryonic Lethality. Cell, 69, 915-926. http://dx.doi.org/10.1016/0092-8674(92)90611-F

[3] Li, E., Beard, C. and Jaenisch, R. (1993) Role for DNA Methylation in Genomic Imprinting. Nature, 366, $362-365$. http://dx.doi.org/10.1038/366362a0

[4] Okano, M., Bell, D.W., Haber, D.A. and Li, E. (1999) DNA Methyltransferases Dnmt3a and Dnmt3b Are Essential for de Novo Methylation and Mammalian Development. Cell, 99, 247-257. http://dx.doi.org/10.1016/S0092-8674(00)81656-6

[5] Fang, M.Z., Wang, Y.M., Ai, N., Hou, Z., Sun, Y., et al. (2003) Tea Polyphenol (-)-Epigallocatechin-3-Gallate Inhibits DNA Methyltransferase and Reactivates Methylation-Silenced Genes in Cancer Cell Lines. Cancer Research, 63, 7563-7570.

[6] Nguyen, C.T., Gonzales, F.A. and Jones, P.A. (2001) Altered Chromatin Structure Associated with Methylation-Induced Gene Silencing in Cancer Cells: Correlation of Accessibility, Methylation, MeCP2 Binding and Acetylation. Nucleic Acids Research, 29, 4598-4606. http://dx.doi.org/10.1093/nar/29.22.4598

[7] Rice, J.C., Massey-Brown, K.S. and Futscher, B.W. (1998) Aberrant Methylation of the BRCA1 CpG Island Promoter Is Associated with Decreased BRCA1 mRNA in Sporadic Breast Cancer Cells. Oncogene, 17, 1807-1812. 
http://www.stockton-press.co.uk/onc http://dx.doi.org/10.1038/sj.onc.1202086

[8] Robertson, K.D., Ait-Si-Ali, S., Yokochi, T., Wade, P.A., Jones, P.L. and Wolffe, A.P. (2000) DNMT1 Forms a Complex with Rb, E2F1 and HDAC1 and Represses Transcription from E2F-Responsive Promoters. Nature Genetics, 25, 338-342. http://dx.doi.org/10.1038/77124

[9] Rountree, M.R., Bachman, K.E. and Baylin, S.B. (2000) DNMT1 binds HDAC2 and a New Co-Repressor, DMAP1, to Form a Complex at Replication Foci. Nature Genetics, 25, 269-277. http://dx.doi.org/10.1038/77023

[10] Clark, S.J. and Melki, J. (2002) DNA Methylation and Gene Silencing in Cancer: Which Is the Guilty Party? Oncogene, 21, 5380-5387. http://dx.doi.org/10.1038/sj.onc.1205598

[11] Hurd, P.J., Whitmarsh, A.J., Baldwin, G.S., Kelly, S.M., Waltho, J.P., Price, N.C., et al. (1999) Mechanism-Based Inhibition of C5-Cytosine DNA Methyltransferases by 2-H Pyrimidinone. Journal of Molecular Biology, 286, 389-401. http://dx.doi.org/10.1006/jmbi.1998.2491

[12] Flotho, C., Claus, R., Batz, C., Schneider, M., Sandrock, I., Ihde, S., Plass, C., Niemeyer, C.M. and Lübbert, M. (2009) The DNA Methyltransferase Inhibitors Azacitidine, Decitabine and Zebularine Exert Differential Effects on Cancer Gene Expression in Acute Myeloid Leukemia Cells. Leukemia, 23, 1019-1028. http://dx.doi.org/10.1038/leu.2008.397

[13] Jahaniani, F., Ebrahimi, S.A., Rahbar-Roshandel, N. and Mahmoudian, M. (2005) Xanthomicrol Is the Main Cytotoxic Component of Dracocephalum kotschyii and a Potential Anti-Cancer Agent. Photochemistry, 66, 1581-1592. http://dx.doi.org/10.1016/j.phytochem.2005.04.035

[14] Masuda, T., Iritani, K., Yonemori, S., Oyama, Y. and Takeda, Y. (2001) Isolation and Antioxidant Activity of Galloyl Flavonol Glycosides from the Seashore Plant, Pemphis acidula. Bioscience, Biotechnology, and Biochemistry, 65, 1302-1309. http://dx.doi.org/10.1271/bbb.65.1302

[15] Huang, H.J., Chen, H.Y., Lee, C.C. and Chen, C.Y. (2014) Computational Design of Apolipoprotein E4 Inhibitors for Alzheimer's Disease Therapy from Traditional Chinese Medicine. BioMed Research International, 2014, 452-625. http://dx.doi.org/10.1155/2014/452625

[16] Morris, G.M., Huey, R., Lindstrom, W., Sanner, M.F., Belew, R.K., et al. (2009) Olson, Auto Dock4 and Auto DockTools4: Automated Docking with Selective Receptor Flexibility. Journal of Computational Chemistry, 30, $2785-2791$. http://dx.doi.org/10.1002/jcc.21256 